\title{
Using Smart Power Management Control to Maximize Energy Utilization and Reliability within a Microgrid of Interconnected Solar Home Systems
}

\author{
Bartosz Soltowski \\ bartosz.soltowski@strath.ac.uk \\ Scott Strachan, \\ Olimpo Anaya-Lara, \\ Damien Frame, \\ University of Strathclyde \\ Glasgow, United Kingdom
}

\author{
Michael Dolan \\ Smarter Grid Solutions \\ Glasgow, United Kingdom
}

\begin{abstract}
Over the past 20 years, off-grid solar home systems (SHS), comprised of solar panels, batteries, a charge controller and loads, have proved the most popular and immediate solution increasing energy access, mainly through rural electrification, across the Global South. Although deployed in significant numbers, issues remain with SHS cost, reliability, utilization and sustainability. Interconnection of SHS to form a microgrid of connected prosumers and consumers may offer a solution that, by employing smart management of the power distribution amongst connected households, has the potential to 'unlock' latent generation and storage capacity and so improve reliability and security of supply, reduce the system cost per head, and ultimately the levelized cost of energy supplied. These factors combine to improve the overall sustainability, efficiency and flexibility of SHS technology. This paper presents the functionality of a Smart Power Management (SPM) that seeks to distribute power across prosumers/consumers connected to a microgrid of interconnected SHS, to maximise the utilisation of available generation and storage across the system and so improve the reliability and affordability of the energy supplied. The SPM is applied and appraised using a simulation involving representative generation and demand profiles for a village with a high penetration of SHS technology. The power management methodology utilizes algorithms applied to manage power flows between customers. The simulated results demonstrate significant improvements in reliability of supply within the microgrid during low generation season.
\end{abstract}

Keywords-Solar Home System, Microgrid, Peer-to-Peer Electricity Trading, Smart Power Management.

\section{INTRODUCTION}

1.2 billion people live without any access to electricity, while a further 1.7 billion are exposed to highly unreliable or unaffordable connections. A significant number of those living without electricity reside in remote rural areas of developing countries with no prospect of connecting to the national power network. To date, off-grid renewable-based systems have presented the most viable means of achieving 'sustainable energy for all'. This could be provided in two ways - by installing microgrids with centralised generation or by using Solar Home Systems which, to date, have proved the most popular and immediate solution, mainly due to efficient market models developed by SHSs distributors and energy service companies (ESCOs). As a result, pay-as-you-go (PAYGO) for SHS market model has attracted more than 220 million USD of investment by the end of 2016 (investment in 2012 was only 3 million USD) [1]. Other factors stimulating investments in off-grid energy section are associated with the falling capital costs for PV modules and energy storage devices. As a result, some African and Asian countries have experienced a rapid growth of SHS technology penetration; for example, at time of writing, more than 4.5 million units have been sold in Bangladesh, and 1.1 million in East Africa [2], [3].

Although SHSs have been very popular in rural areas of developing countries, problems with matching appropriate SHS size to customers' needs remain. According to SHS providers, significant numbers of SHS users soon after having their systems installed, request more electricity than the system is capable of generating [4]. Simultaneously, investment in SHS requires users to pay for the technology regular payments which can be difficult for families in these rural settings living on incomes around the international poverty line of $\$ 2$ per person per day [5].

Innovation that addresses these issues and considers the role of SHS in delivering cost efficient and scalable energy access solutions is required. Interconnection of SHS to form a microgrid of connected prosumers and consumers is a solution that, by employing Smart Power Management of the power distribution amongst connected households enabling P2P energy exchange and business models, has the potential to balance supply and demand within the microgrid, offering consumers more affordable energy, and energy providers a greater return on their investment.

All these principles are applied to a case-study village consisting of 12 interconnected SHSs, forming a microgrid to enable power sharing between prosumers. The developed SPM control strategy seeks to accommodate different types of customer connection scenarios, with the objective of maintaining a satisfactory level of supply reliability, while also providing the flexibility required to manage the 
affordability of energy provided to customers with different needs, and so making the microgrid more sustainable.

\section{SMART POWER MANAGEMENT}

Each customer connected to the proposed microgrid is equipped with a PV module, battery and charge controller. Battery storage capacity in SHSs is required to ensure an electrical supply is available in the evenings when zero solar resource exists for generation, and to offer days of autonomy during prolonged periods of low solar resource. However, in an interconnected SHS microgrid arrangement, gives users the flexibility to import and export energy and maximize the available pooled storage (and generation) capacity. The Smart Power Management has been designed to optimally manage the pooled generation and storage capacity, by dynamically controlling individual power import/export transactions between connected customers based on their current demand requirements and available storage capacity and generation output.

In order to define how much electricity needs to be traded between users it is essential to identify power consumption patterns by each customer connected to the network. The proposed SPM controller has a predefined consumption profiles in order to estimate when SHS users are likely to have an energy surplus and deficit. Based on the derived patterns as well as on bids and offers among other users connected to the microgrid, the SPM controller makes decisions on whether to import or export electricity, how it should be distributed and at what cost.

SolShare is the only technology [6] connecting Solar Home Systems to form a microgrid and enabling power exchange between SHSs using a control strategy that relies on the use of a central hub to equalize the distribution of power between users willing and able to buy electricity from the local energy market. However, while this does attempt to utilize latent generation, storage capacity, the technology does not optimize power flows while respecting distances between importers and exporters. As a consequence of that, losses in the microgrid may be significantly high. The proposed SPM controller described in this paper allows users having SHSs to connect with neighbors directly (peer to peer) or indirectly (peer to peer to peer), but avoids the need to route power through a central hub to ensure an equal distribution of power across customers willing to import. The scalability of this control strategy means that the physical expansion of the microgrid, required to meet the inevitable demand growth, is much easier. The proposed SPM controller system builds locational pricing for energy at each household connected to the microgrid based on pre-defined distribution line parameters. As a consequence, it prioritizes power transfers between customers who are directly interconnected over those who are located some distance from each other, with no direct connection. An additional benefit of applying SPM control is the increased system redundancy. Failure of a single SPM controller or distribution line, will not affect the microgrid operation, as it can continue to operate as two or more islanded networks.
This is a particularly important aspect for microgrids built in developing countries where accessibility to the network is limited and maintenance of the system difficult, resulting in prolonged outage periods.

Another important feature of the SPM control strategy is the use of variable prosumer energy tariffs to encourage buyers to import electricity at the periods of high generation - when the cost of energy is at its lowest. For net exporters, cost of energy offered will be the lowest at periods of high generation and low demand in order to attract customers to import electricity which would not otherwise be utilized.

\section{MICROGRID MODEL TOPOLOGY}

This section of the report presents topography of an interconnected village considered in this paper. Connections between customers are illustrated in the Fig. 1 where each SHS is linked to the rest of the microgrid using at least one connection. Two biggest prosumers are located in the central location of the village (SHS1 and SHS2). Each of them is connected to three other SHSs and has a capability to pass offers and bids between SHSs which are not necessarily directly connected between adjacent neighbors (for example between SHS 2 and SHS 10). This information is carried respecting distribution losses within the system.

All connections within the village were set to have the same resistance to demonstrate how the algorithm optimizes power flows at each location of the network.

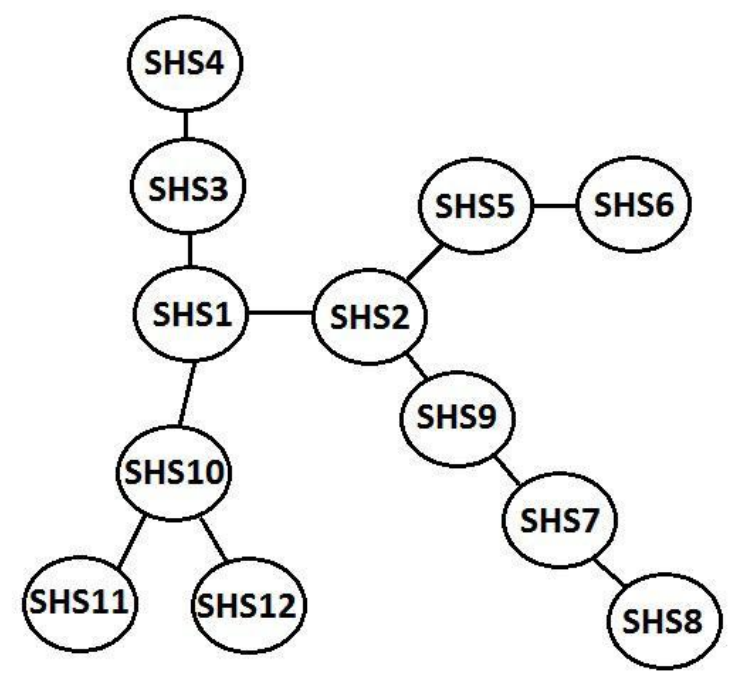

Figure 1. Topology of Analyzed Microgrid

The topography of the village illustrated in the Fig. 1 is used to generate results illustrating differences in reliability of supply between interconnected and non-interconnected SHSs. It was also estimated how much energy would be transferred to, and from, each of the household during low generation periods. 


\section{MICROGRID MODEL DEMAND DISTRUBUTION}

Smart Power Management simulations were performed on a village consisting of 12 SHSs. Models were developed using Matlab Simulink and represent the power flows between and import/export behavior of interconnected Solar Home Systems controlled by the proposed SPM controller. The model was primarily designed to investigate how interconnection of SHSs enabling power exchange between users can enhance the reliability of supply in a microgrid arrangement. The model represents SHSs with a typical generation capacity ranging from $30 \mathrm{~W}$ up to $50 \mathrm{~W}$, which are typical capacities of SHSs deployed in Bangladesh. Demand patterns were generated separately for each SHSs and are repeatable daily for use in the simulation [7]. These profiles were generated using LoadProGen [8] and are representative of typical SHS installations in Bangladesh where the technology is prevalent, providing $11 \%$ of the population with access to electricity.

The capacities and demands vary for each SHS user. Ten of SHS demand profiles used in the simulation represent domestic customers, while the remaining two (SHS1 and SHS2) represent commercial users (e.g. productive uses of energy [9] such as shops, barbers, small businesses, etc) requiring significantly higher electricity demand. In this instance a base demand of $25 \mathrm{~W}$ representing a small DC fridge is considered.

The first group of domestic customers is assumed to use $50 \mathrm{~W}$ SHSs. These customers typically consume electricity in the morning and late afternoon. The most commonly used devices by these customers are light bulbs, radios and TVs. The average daily demand typical of a 50W SHS installed in Bangladesh is illustrated in the Table I.

\begin{tabular}{|c|c|c|c|}
\hline Appliance & Power (W) & Time (h) & Energy (Wh) \\
\hline L. Bulb 1 & 6 & 5 & 30 \\
L. Bulb 2 & 6 & 4 & 24 \\
L. Bulb 3 & 6 & 2 & 12 \\
Radio & 10 & 1 & 10 \\
TV & 20 & 4 & 80 \\
\hline Total & - & - & 156 \\
\hline \multicolumn{4}{|c|}{ Table I. Typical Demand for 50W SHS. }
\end{tabular}

A Matlab Simulink model designed to show the behavior of the SPM controlled system considers six SHSs customers using $50 \mathrm{~W}$ installations. According to Table I, the total average daily demand typical of this group is $156 \mathrm{Wh}$. In order to present how SHS customers can rely on each other using SPM, demand presented in Table I was diversified for each user. As such, it is assumed that two 50W SHSs customers require $30 \%$ more electricity than previously specified typical of 50W SHS usage. This group of users will rely on electricity imported from their neighbors. Another two prosumers with $50 \mathrm{~W}$ SHS consume $20 \%$ less than the average daily demand specified in Table I, and so have a net surplus of electricity, enabling them to share their energy with customers consuming larger amounts of energy than they can generate or have available in their local battery storage. The remaining $50 \mathrm{~W}$ SHSs users are 'typical' users, consuming $156 \mathrm{Wh}$ per day i.e. typical average demand for this size of SHS. Although, most of the time, this group of customers generates sufficient energy to satisfy their own needs, they can experience problems during periods of low resource/generation, when their demand may exceed generation and available storage.

Another group of SHSs connected to the microgrid uses 40W systems. Typical demand for this group of customers is lower than that associated with the 50W SHSs previously considered, mainly due to a lower number of appliances being used as well as a shorter operating time associated with these appliances. The average demand for electricity for the $40 \mathrm{~W}$ SHS is $124 \mathrm{Wh}$ as sown in Table II, which presents typical appliances, daily demand and operating times for this group of customers.

\begin{tabular}{|c|c|c|c|}
\hline Appliance & Power (W) & Time (h) & Energy (Wh) \\
\hline L. Bulb 1 & 6 & 5 & 30 \\
L. Bulb 2 & 6 & 4 & 24 \\
Radio & 10 & 1 & 10 \\
TV & 20 & 3 & 60 \\
\hline Total & - & - & 124 \\
\hline \multicolumn{4}{|c}{ Table II. Typical Demand for 40W SHS }
\end{tabular}

Again, demand at each 40W SHS installation was diversified in order to illustrate how much energy could be transferred between sellers and buyers connected to the same microgrid. One of the 40W SHSs customers was modelled to consume an average of $124 \mathrm{Wh}$ (average demand according to Table II), while two other households were modelled to consume $20 \%$ more than average $40 \mathrm{~W}$ system and $15 \%$ less electricity than average $40 \mathrm{~W}$ demand.

In addition to that, one of the SHSs in the village was modelled as a 30W SHS with average generation of $126 \mathrm{Wh}$ and daily demand of $104 \mathrm{Wh}$. Electrical demand for $30 \mathrm{~W}$ SHS was presented in the Table III.

\begin{tabular}{|c|c|c|c|}
\hline Appliance & Power (W) & Time (h) & Energy (Wh) \\
\hline L. Bulb 1 & 6 & 5 & 30 \\
L. Bulb 2 & 6 & 4 & 24 \\
Radio & 10 & 1 & 10 \\
TV & 20 & 2 & 40 \\
\hline Total & - & - & 104 \\
\hline \multicolumn{4}{|c|}{ Table III. Typical Demand for 30W SHS }
\end{tabular}

Demand for electricity was constant, independently of the generation.

Fig. 2 illustrates each SHS connected to the local microgrid, its daily energy balance at the average capacity factor in Bangladesh - $17.6 \%$ (blue) as well as at the capacity factor representing low generation season $-12.6 \%$ (red). 


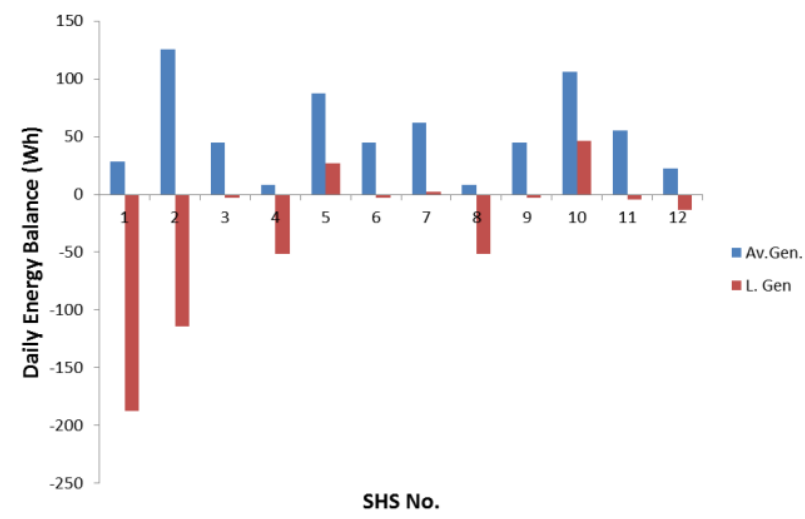

Figure 2. Daily Energy Balance for each SHS Connected to the Microgrid at the Average and Low Capacity Factor

According to Fig. 2, all households within the village at the average generation capacity factor have an energy surplus. As a result, nobody connected to the microgrid is required to buy or sell electricity on the local pool. Problems with power shortages may arise during low generation periods and may have a negative impact on users with relatively high demands in comparison to generation, such as SHS 1, SHS 2, SHS 4, SHS 8. Despite low generation periods, users can import electricity while their system experience minor technical issues associated with the failure of their own PV module or energy storage. Problems related to power shortages could be minimized by imports of power from other users connected to the microgrid, who have an energy surplus. By applying the proposed SPM control strategy, appropriate amounts of electricity are exchanged between exporters and importers to ensure all demand is met as optimally as possible. In doing so, customers with a surplus of energy gain benefits by selling electricity, while those with an energy deficit can import energy and increase their average supply availability (reduce their loss of load probability and cumulative time off-supply). The optimal rate of energy to sell is defined by the proposed SPM controller such that the system always retains a minimum energy reserve for each seller, while meeting buyers' energy requirements whenever possible.

\section{RESULTS}

This section compares the results of simulations run for an interconnected microgrid of SHS systems village with those obtained from the simulation of the same SHS distribution without interconnection, using the generation and demand data specified in Fig. 2. The simulation shows results for a one month period with an average generation capacity factor of $12.6 \%$ and a daily generation standard deviation of $2.62 \%$. The initial state of charge of each SHS battery is set to $75 \%$. The battery capacities were defined according to requirements specified in [10], and set to maximum allowed capacities defined by the formula 1 .

$$
\operatorname{Capacity}(A h)=P_{P V}(W) \times 1.5
$$

Where, Capacity - represents size of energy storage, $\mathrm{P}_{\mathrm{PV}}$ peak power production by the SHS.

Based on (1), 30W SHS was modelled to use 45Ah batteries, 40W SHS as $60 \mathrm{Ah}, 50 \mathrm{~W}$ SHS as $75 \mathrm{Ah}, 180 \mathrm{~W}$ as $270 \mathrm{Ah}$, $200 \mathrm{~W}$ as 300Ah. Typical battery technology used for SHS is a lead-acid, therefore, minimum recommended state of charge of the battery is set to $50 \%$.

Based on explained assumptions simulation was run. Fig. 3 illustrates differences between the average time without power per day for non-interconnected (stand-alone) SHSs and an interconnected SHS microgrid system.

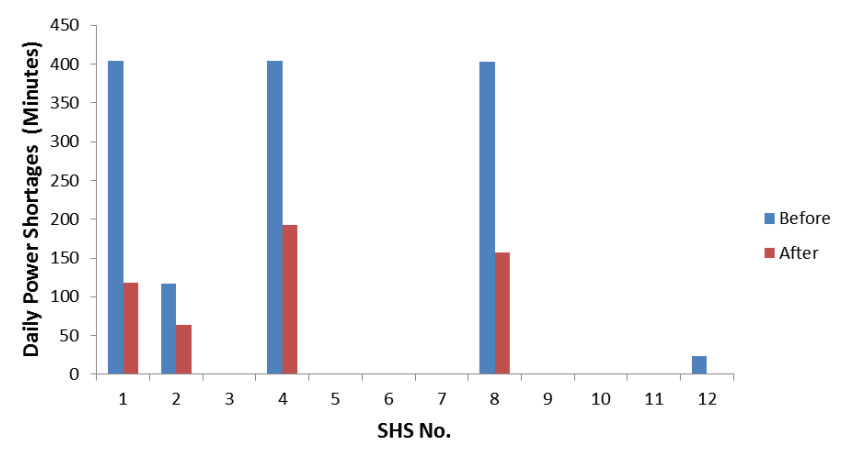

Figure 3. Differences in Reliability of Supply for a Stand-alone and Microgrid Systems

In order to present functionality of the algorithm according to which SPM controls power flows within the microgrid, it is required to generate data showing which SHSs imported how much electricity and which of them sold it. This is illustrated in the Fig. 4 presenting total monthly energy import (positive) and export (negative) for each SHS connected to the microgrid.

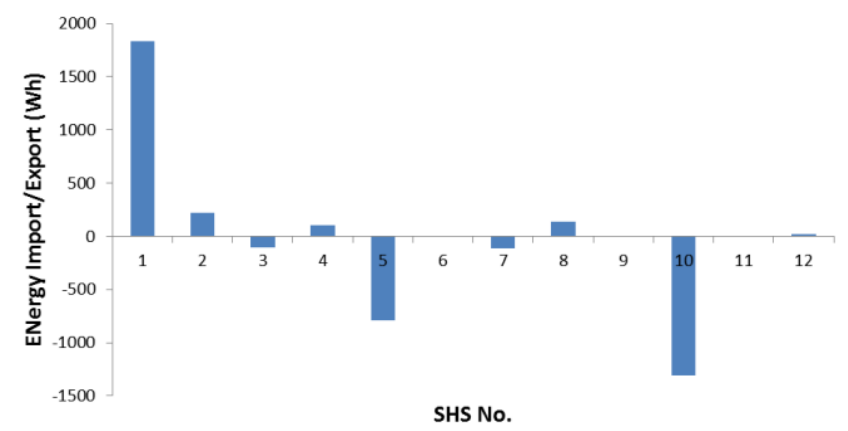

Figure 4. Monthly Energy Import/Export (Wh) 


\section{ANALYSIS}

Fig. 3 shows significant improvements in reliability of supply for the interconnected microgrid system. The meantime without power per household connected to this microgrid during low generation season was reduced by around $60 \%$ from that seen for the same village using noninterconnected, stand-alone SHSs. The results show that the greatest increase in reliability of supply was obtained by customers directly connected to net electricity exporters, such as when SHS 1 imports power from SHS 10, or SHS 4 buying electricity from SHS 3. By analyzing Fig. 4 it can be noticed that customers with a significantly larger generation than demand exported electricity to net power importers not directly connected to them. This is well illustrated by SHS 5 which exported 792 Wh of energy within the whole 30-day period. SHS 5 is directly connected only to SHS 6 and SHS 2 which in total imported $220.5 \mathrm{Wh}$, meaning that the remaining $571.5 \mathrm{Wh}$ was exported to other customers within the microgrid which require additional energy. SHS 6, 9 and 11 were passive while running the simulation. There was no requirement to import electricity for those customers as they generated sufficient amount to satisfy their demand. Simultaneously, these users did not produce sufficient amount of energy to become competitive on the local energy market among energy exporters.

SPM system also assured prosumers with a net energy export (SHS3, 5, 7, 10) to keep a reserve required for backup electricity needs by each system. As a result, none of energy exporters experienced any power shortage during the low generation period analysed.

\section{FUTURE WORK}

Results generated for this paper are based on a village which demand and supply were estimated using literature review. In order to present full potential of a Smart Power Management for interconnected SHSs microgrids it is required to gather real data on demand and generation at the particular village with high density of SHSs installations and run simulations which will present real case study village.

Other important feature required in the model is addition of technical losses during power conversion. Simulink model developed so far does not include efficiencies of power converters as well as energy storage devices. They are modelled as systems with $100 \%$ efficiency. In order to make sure that the model does not charge/discharge batteries too fast it is required to develop appropriate capacity management system.

Further work will also require studies related to finding threshold points for installations of these types of microgrids depending on demand diversity in a village. For locations where consumption patterns for each SHS user are similar rate of energy exchanged between prosumers could be very low and SHSs would not support each other in periods of low power generation.

Other aspects required to be analyzed consider faults on the distribution system and capability to separate system into two or more islanded microgrids. This could significantly improve reliability of power supply within the off-grid environment.

Other studies will include analysis of different network configurations for proposed microgrids. It is important to connect SHS users with a minimum use of distribution lines in order to reduce investment costs of a distribution lines.

Creating a local energy pool could also be a solution for families which currently do not have access to electricity. Surplus of energy generated by SHSs in the village could be used to provide energy for a number of customers who do not currently have SHSs. Future work will include analysis which will define requirements for connections for those kind of microgrid users.

\section{REFERENCES}

[1] C. Lins, "Renewables 2017 Global Status Report", Renewable Energy Policy for the 21st Century, 14 June 2017

[2] T.Buckley, S.Nicholas, S.J Ahmed, "Bangladesh Electricity Transition: A Diverse, Secure and Deflationary Way Forward", Institute for Energy Economics and Financial Analysis, November 2016

[3] "2Q 2017 Frontier Power Market Outlook - Micro-grids on the horizon”, Bloomberg New Energy Finance, 25 April 2017

[4] "How we made it in Africa", "Four things to know about Africa's pay-as-you-go solar energy market”, 2016. [Online]. Available: https://www.howwemadeitinafrica.com/four-things-knowafricaspay-go-solar-energy-market/. [Acessed: 24- June- 2017]

[5] "Could you like on $1.90 \$$ a day?", J.Hickel, [Online], Available: https://www.theguardian.com/global-development-professionalsnetwork/2015/nov/01/global-poverty-is-worse-than-you-thinkcould-you-live-on-190-a-day. [Accessed: 24-June-2017]

[6] P. Hollberg, "Swarm Grids - Innovation in rural electrification", MSc Thesis, KTH School of Industrial Engineering and Management, Sweden, 2015

[7] "Performance of Solar Home Systems in Rural Bangladesh", S.A. Chowdhury, S.M. Raiyan Kabir, Department of EEE, United International University, Dhaka, Bangladesh.

[8] F. Rica, M. Monacecchi, M. Merlo, "Novel procedure to formulate load profiles for off-grid rural areas - the Load Pro Gen software", Politecnico di Milano

[9] GSMA, "Distribution of Solar Pay-as-you-go in Pakistan", EcoEnergyFinance, 2016, pp. 30-31

[10] "Technical Specification for Solar Home System (SHS)", Idcol Solar Programe, March 2017, pp. 10-11 